\title{
Body composition assessment of vegetarian-vegan and omnivore young women - an exploratory study
}

\author{
Avaliação da composição corporal de mulheres jovens vegetarianas e omnívoras - \\ um estudo exploratório
}

\author{
Cíntia Ferreira-Pêgo ${ }^{1 *}$, Rejane Giacomelli Tavares ${ }^{1}$, Sofia Lopes ${ }^{2}$, Tatiana Fontes ${ }^{1}$, Luís Monteiro Rodrigues ${ }^{1}$ \\ ${ }^{1}$ CBIOS - Universidade Lusofona's Research Center for Biosciences \& Health Technologies, Campo Grande 376, 1749-024 \\ Lisboa, Portugal \\ ${ }^{2}$ School of Health Sciences and Technologies, Universidade Lusófona de Humanidades e Tecnologias, Lisboa, Portugal \\ *corresponding author: cintia.pego@ulusofona.pt
}

\begin{abstract}
Diet is commonly accepted as a determinant of body composition, especially when related to specific lifestyles. Vegetarian-vegan diets, which involve a reduction or elimination of animal product consumption, are believed to be more "healthy," facilitating weight control and reducing the incidence and clinical course of different diseases, in particular those related to overweight and obesity. Global reviews and metanalysis on these issues, however, are still insufficient. Our preliminary approach addresses the total body composition differences among vegetarians-vegans and omnivorous individuals. This cross-sectional study involved ten healthy women, five vegetarian-vegan, and five omnivores (mean 28.10 years old). Body composition was assessed using a dual-energy x-ray absorptiometry (DXA Lunar Prodigy Advance - General Electric Healthcare ${ }^{\circledR}$ ). Other general and sociodemographic variables were also collected by trained dietitians. Our results have shown that the vegetarian-vegan group had a non-significantly lower volume of all types of body mass (total bone, fat, lean, tissue, and fat-free) evaluated. Additionally, the vegetarian-vegan group presented higher values of visceral adipose tissue and subcutaneous adipose tissue $\left(286.20\right.$ and $11138.40 \mathrm{~cm}^{3}$, respectively, $p>$ 0.05 ) compared to the omnivore group. These differences will be further confirmed in subsequent studies.
\end{abstract}

Keywords: Body composition; visceral adipose tissue; subcutaneous adipose tissue; vegetarian-vegan diet; Dual-energy $\mathrm{x}$-ray absorptiometry

\footnotetext{
Resumo

A dieta é geralmente aceite como determinante da composição corporal, especialmente quando relacionada com estilos de vida específicos. Acredita-se que as dietas vegetarianas-veganas que envolvem uma redução ou eliminação do consumo de produtos animais, são mais "saudáveis" facilitando o controlo do peso e reduzindo a incidência e o curso clínico de diferentes doenças. No entanto, as revisões e meta-análises sobre estas questões são ainda insuficientes. Anossa abordagem preliminar aborda as diferenças na composição corporal total entre vegetarianos-vegan e indivíduos omnívoros. Este estudo transversal envolveu dez mulheres saudáveis, cinco vegetarianas-veganas, e cinco omnívoras (média de 28,10 anos de idade). A composição corporal foi avaliada utilizando uma absorptiometria de raios X de dupla energia (DXA Lunar Prodigy Advance - General Electric Healthcare ${ }^{\circledR}$ ). Outras variáveis gerais e sociodemográficas foram também recolhidas por nutricionistas treinados. Os resultados mostraram que o grupo vegetariano-vegan teve um volume inferior não significativo de qualquer tipo de massa (osso, gordura, massa magra, tecido, tecido livre de gordura) avaliada. Além disso, estas mulheres jovens vegetarianas-vegan apresentaram valores mais elevados de tecido adiposo visceral e tecido adiposo subcutâneo (286,20 e 11138,40 $\mathrm{cm}^{3}$, respetivamente, $\left.p>0,05\right)$ em comparação com o grupo omnívoro. Estas diferenças serão ainda confirmadas em estudos subsequentes.
}

Palavras-chave: Composição corporal; tecido adiposo visceral; tecido adiposo subcutâneo; dieta vegetariana; absorptiometria de raios $\mathrm{X}$ de dupla energia 


\section{Introduction}

Diet seems to have a significant influence on the development of adiposity, obesity, and cardiometabolic diseases (1). It is also clear that excess body fat is an established risk factor for many disease conditions and premature death (2).

Plant-based diets such as vegetarianism and veganism are believed to reduce body weight because of the overall decreased caloric intake and increased thermogenesis. These diets are most simply defined by a reduction or elimination of the consumption of animal products and based on the consumption of grains, legumes, vegetables, fruits, and nuts (3). However, it should be noted that reductions in body weight do not necessarily result in reductions in adipose tissue neither directly translate into improved health outcomes (4). Nevertheless, the vegetarian diet is reported as being an effective means for the prevention and treatment of cardio-metabolic diseases (3). The Academy of Nutrition and Dietetics, the world's largest organization of food and nutrition professionals founded in 1917, recognizes the health and nutritional benefits for the prevention and treatment of certain diseases potentially related to vegetarian-vegan diets (5). Healthy, properly planned vegetarian diets were described as effective for glycemic control while providing metabolic and cardiovascular benefits, including the control of atherosclerosis dyslipidemia and blood pressure (3). These cardio-metabolic benefits were reported to be greater with vegan than lacto-ovo-vegetarian diets (6). However, the body composition of vegetarian and vegan individuals has not been sufficiently studied, and commonly-used assessments of waist circumference, body weight, or Body Mass Index (BMI) do not reveal body composition nor fat distribution. Therefore, the main goal of the present study was to approach and compare the body composition - bone mass, fat mass, lean mass, tissue mass, fat-free mass, total mass, and visceral and subcutaneous adipose tissue - of young healthy adult women with different diet regimes (vegetarian-vegan vs. omnivore) by referenced methods.

\section{Introdução}

A dieta parece ter uma influência significativa no desenvolvimento da obesidade e das doenças cardiometabólicas (1). É também evidente que o excesso de gordura corporal é um fator de risco estabelecido para muitas doenças e até para a morte prematura (2).

Acredita-se que as dietas baseadas em plantas como o vegetarianismo e o veganismo reduzem o peso corporal devido à diminuição global da ingestão calórica e ao aumento da termogénese. Estas são basicamente definidas por uma redução ou eliminação do consumo de produtos animais, sendo baseadas no consumo de grãos, legumes, vegetais, frutas e frutos secos (3). Contudo, é de notar que as reduções no peso corporal não resultam necessariamente em reduções no tecido adiposo nem se convertem diretamente em melhores resultados para a saúde (4). No entanto, a dieta vegetariana é relatada como sendo um meio eficaz para a prevenção e tratamento de doenças cardiometabólicas (3). A Academia de Nutrição e Dietética, a maior organização mundial de profissionais de alimentação e nutrição fundada em 1917, reconhece os benefícios para a saúde e nutrição para a prevenção e tratamento de certas doenças potencialmente relacionadas com as dietas vegetarianas (5). Dietas vegetarianas saudáveis e devidamente planeadas foram descritas como eficazes para o controlo glicémico, proporcionando ao mesmo tempo benefícios metabólicos e cardiovasculares, incluindo o controlo da dislipidemia e da pressão arterial (3). Estes benefícios cardiometabólicos foram reportados como sendo maiores com as dietas veganas do que as ovo-lacto-vegetarianas (6). Contudo, a composição corporal dos indivíduos vegetarianos e veganos não é suficientemente estudada, tendo também em conta que as avaliações da circunferência da cintura, peso corporal, ou Índice de Massa Corporal (IMC) não revelam a composição corporal nem a distribuição da gordura. Portanto, o principal objetivo do presente estudo foi abordar e comparar a composição corporal - massa óssea, massa gorda, massa magra, massa tecidual, massa sem gordura, massa total, e tecido adiposo visceral e subcutâneo, de jovens mulheres adultas saudáveis com diferentes regimes alimentares (vegetarianos-veganos vs. omnívoros) através de métodos referenciados. 


\section{Materials and Methods}

\section{Study design and participants}

The present study was an observational, quantitative, and cross-sectional analysis designed to evaluate total body composition in vegetarian-vegan individuals and comparing it with omnivore diet participants.

A convenience sample of ten participants (five vegetarian-vegan, and five omnivores) all women presenting a mean age of 28.10 (SD: 6.94) years old were selected from the university's student body. Participants were selected following previously defined inclusion/ non-inclusion criteria. Apart from gender, non-inclusion criteria included any kind of disease or health alteration, taking any sort of medication, being pregnant or breastfeeding, and being in the first week of their menstrual cycle. All participants agreed to participate in the study, giving their informed written consent. Procedures respected the principles of good clinical practice adopted for human research studies complying with good clinical practice ethical standards for human research (7). Participants' sociodemographics (age, course year, residence, and other lifestyle habits) were obtained and a Food Frequency Questionnaire and a 3-day dietary recall were also collected for each participant.

\section{Body composition assessment}

Weight was measured on an electronic scale $[0.1 \mathrm{~kg}$ (0.1-200 kg) accuracy], wearing light clothing and no shoes. Height was a self-reported variable used to calculate BMI by the formula [BMI = weight $(\mathrm{kg}) /$ height $\left.\left(\mathrm{m}^{2}\right)\right]$.

The Dual-energy x-ray absorptiometry (DXA Lunar Prodigy Advance - General Electric Healthcare ${ }^{\circledR}$; Chicago, Illinois, USA) was used to measure bone mass, fat mass, lean mass, tissue mass, fat-free mass, total mass, and visceral and subcutaneous adipose tissue. Before each whole-body scan, DXA was calibrated according to the manufacturer's instructions via a standard calibration block. Measurements were performed under at least four hours of fasting conditions and without exercise the previous 24 hours to ensure adequate hydration conditions. Participants removed shoes, socks, and all jewelry. Wearing underwear or close-fitting clothing with no metallic piecess, participants were instructed to lie supine on the scanning bed with hands by their sides, not touching the body. During all body scans, participants were asked to remain motionless, and Velcro straps were situated

\section{Material e Métodos}

\section{Desenho do estudo e participantes}

O presente estudo foi uma análise observacional, quantitativa e transversal concebida para avaliar a composição corporal total em indivíduos vegetarianosveganos e compará-la com os participantes omnívoros.

Uma amostra de conveniência de dez participantes (cinco vegetarianos-veganos, e cinco omnívoros) todas mulheres com uma idade média de 28,10 (DP: 6,94 ) anos foram selecionadas do corpo estudantil da universidade. Os participantes foram selecionados de acordo com critérios de inclusão/ não-inclusão previamente definidos. Para além do sexo, os critérios de não-inclusão incluíam qualquer tipo de doença ou alteração de saúde, tomar qualquer tipo de medicação, estar grávida ou a amamentar, e estar na primeira semana do seu ciclo menstrual. Todos os participantes concordaram em participar no estudo, dando o seu consentimento informado por escrito. Os procedimentos respeitaram os princípios de boas práticas clínicas adotados para estudos de investigação em humanos, cumprindo as normas éticas de boas práticas clínicas para a investigação em humanos (7). Foram obtidos os dados sociodemográficos dos participantes (idade, ano de curso, residência, e outros hábitos de vida) e foram também recolhidos dados alimentares mediante um Questionário de Frequência Alimentar um diário alimentar de 3 dias para cada participante.

\section{Avaliação da composição corporal}

$\mathrm{O}$ peso foi medido numa balança eletrónica $[0,1 \mathrm{~kg}$ (0,1-200 kg) de precisão], usando roupas leves e sem sapatos. A altura foi uma variável auto reportada utilizada para calcular o IMC através da fórmula [IMC $=$ peso $(\mathrm{kg}) /$ altura $\left.\left(\mathrm{m}^{2}\right)\right]$.

A absorptiometria de raios $\mathrm{X}$ de dupla energia (DXA Lunar Prodigy Advance - General Electric Healthcare ${ }^{\circledR}$; Chicago, Illinois, EUA) foi utilizada para medir massa óssea, massa gorda, massa magra, massa de tecido, massa livre de gordura, massa total, e tecido adiposo visceral e subcutâneo. Antes de cada scan de corpo inteiro, o DXA foi calibrado de acordo com as instruções do fabricante através de um bloco de calibração padrão. As medições foram realizadas sob pelo menos quatro horas de jejum e sem exercício nas 24 horas anteriores para assegurar condições de hidratação adequadas. Os participantes removeram sapatos, meias, e todas as joias, vestiram roupa interior, ou roupa apertada sem partículas de metal, e foram instruídos a deitar-se em 
around the ankles and knees. All participants on the DXA scanning bed were positioned on the scanning bed by the same trained researcher. Scans lasted approximately 5 to 10 minutes. The researcher analyzed each scan to adjust software-determined regions of interest before producing the whole-body report.

\section{Statistical analysis}

Data were presented either as means and standard deviation (SD) for continuous variables or percentages and absolute frequencies for dichotomous variables. The normality of the variables was tested. As all followed a normal distribution, the distribution of the selected characteristics between groups was tested using $\chi^{2}$ tests for categorical variables or Student's t-tests for continuous variables. All statistical tests were two-tailed and the significance level was set at $p<0.05$. All analyses were performed using the SPSS software version 27.0 (SPSS Inc, Chicago, IL, USA).

\section{Results}

The general characteristics of the studied population according to its dietary pattern are summarized in Table 1. Omnivorous women were statistically taller than the vegan-vegetarian group, however, this difference does not affect BMI since no other differences in body weight were found. Sleeping hours were also statistically different between the groups analyzed, as participants following an animal-product diet slept fewer hours compared to the vegetarian group. No other statistically relevant differences were found.

The total population presented a mean bone mass of $2285.30 \mathrm{~g}, 18983.60 \mathrm{~g}$ of fat mass, $40532.60 \mathrm{~g}$ of lean mass, $59516.10 \mathrm{~g}$ of tissue mass, $42817.80 \mathrm{~g}$ of fat-free mass, and finally $63.40 \mathrm{~kg}$ of total body mass. Vegetarian-vegan women presented lower volume of all body mass types. However, none of these differences were statistically significant. The results are shown in Figure 1. posição supino no leito de varredura com as mãos pelos lados, não tocando o corpo. Durante todas as varreduras do corpo, foi pedido aos participantes que permanecessem imóveis, enquanto as tiras de velcro se situavam à volta dos tornozelos e joelhos. As varreduras duraram aproximadamente 5 a 10 minutos. O mesmo investigador posicionou todos os participantes no leito de varrimento DXA. Além disso, o investigador treinado analisou cada varredura para ajustar as regiões de interesse determinadas pelo software antes de produzir o relatório de corpo inteiro.

\section{Análise estatística}

Os dados foram apresentados como médias e desvio padrão (DP) para variáveis contínuas ou percentagens e frequências absolutas para variáveis dicotómicas. A normalidade das variáveis foi testada, e todas elas seguiram uma distribuição normal, por este motivo, a distribuição das características selecionadas entre grupos foi testada utilizando os testes $\chi^{2}$ para variáveis categóricas ou os testes $t$ de Student para variáveis contínuas. Todos os testes estatísticos foram bi-caudais e o nível de significância foi fixado em $\mathrm{P}<0,05$. Todas as análises foram realizadas utilizando o software SPSS versão 27.0 (SPSS Inc, Chicago, IL, EUA).

\section{Resultados}

As características gerais da população estudada de acordo com o seu padrão dietético estão resumidas na Tabela 1. As mulheres omnívoras foram estatisticamente mais altas em comparação com o outro grupo analisado, contudo, esta diferença não afeta o IMC uma vez que não foram encontradas outras diferenças no peso corporal. As horas de sono também foram estatisticamente diferentes entre os dois grupos analisados, uma vez que os participantes que seguem uma dieta de origem animal dormiram menos horas em comparação com o grupo vegetariano. Não foram encontradas outras diferenças estatisticamente relevantes.

A população total apresentou uma massa óssea média de 2285,30 g, 18983,60 g de massa gorda, 40532,60 g de massa magra, 59516,10 g de massa tecidual, 42817,80 $\mathrm{g}$ de massa sem gordura, e finalmente $63,40 \mathrm{~kg}$ da massa total. As mulheres vegetarianas-vegetarianas apresentaram um volume mais baixo de todo o tipo de massa corporal. No entanto, nenhuma destas diferenças foi estatisticamente significativa. Estes resultados podem ser observados na Figura 1. 
Table 1 - General characteristics of the population according to dietary pattern.

Tabela 1 - Características gerais da população de acordo com o padrão alimentar.

\begin{tabular}{|c|c|c|c|c|}
\hline & $\begin{array}{c}\text { Total population/ } \\
\text { População total } \\
(n=10) \\
\end{array}$ & $\begin{array}{c}\text { Omnivore/ } \\
\text { Omnívoros } \\
(\mathbf{n}=5) \\
\end{array}$ & $\begin{array}{c}\text { Vegetarian-Vegan/ } \\
\text { Vegetarianos-Vegan } \\
(\mathrm{n}=5) \\
\end{array}$ & $p$-value ${ }^{a}$ \\
\hline Age, years / Idade, anos & $28.10(6.94)$ & $25.60(4.56)$ & $30.60(8.47)$ & 0.054 \\
\hline Height, m / Altura, m & $1.65(0.06)$ & $1.68(0.02)$ & $1.61(0.07)$ & 0.003 \\
\hline Weight, kg / Peso, kg & $61.78(6.81)$ & $64.30(6.60)$ & $59.26(6.71)$ & 0.847 \\
\hline BMI, $\mathrm{kg} / \mathrm{m}^{2} / \mathrm{IMC}, \mathrm{kg} / \mathrm{m}^{2}$ & $22.75(1.48)$ & $22.75(1.75)$ & $22.76(1.36)$ & 0.938 \\
\hline Smoking habits / Hábito tabágico, \% (n) & $30.00(3)$ & $40.00(2)$ & $20.00(1)$ & 0.490 \\
\hline $\begin{array}{l}\text { Practice of physical exercise / } \\
\text { Prática de exercício físico, \% (n) }\end{array}$ & $90.00(9)$ & $100.00(5)$ & $80.00(4)$ & 0.292 \\
\hline Sleep, hours / Sono, horas & $7.28(1.12)$ & $6.38(1.18)$ & $8.00(0,00)$ & 0.014 \\
\hline $\begin{array}{l}\text { Year of course, years / } \\
\text { Ano do curso, anos }\end{array}$ & $2.70(1.42)$ & $2.80(1.64)$ & $2.60(1.34)$ & 0.515 \\
\hline $\begin{array}{l}\text { Working student / } \\
\text { Trabalhador-estudante, \% (n) }\end{array}$ & $30.00(3)$ & $40.00(2)$ & $20.00(1)$ & 0.490 \\
\hline \multicolumn{5}{|l|}{$\begin{array}{l}\text { Environment of residence / } \\
\text { Ambiente de residência, \% (n) }\end{array}$} \\
\hline $\begin{array}{l}\text { Rural / Rural } \\
\text { Urban / Urbano }\end{array}$ & $\begin{array}{l}20.00(2) \\
80.00(8)\end{array}$ & $\begin{array}{l}20.00(1) \\
80.00(4)\end{array}$ & $\begin{array}{l}20.00(1) \\
80.00(4)\end{array}$ & 1.000 \\
\hline \multicolumn{5}{|l|}{$\begin{array}{l}\text { Monthly family income / } \\
\text { Rendimentos familiars mensais, \% (n) }\end{array}$} \\
\hline $\begin{array}{l}<1000 € \\
>1000 €\end{array}$ & $\begin{array}{l}22.20(2) \\
77.80(7)\end{array}$ & $\begin{array}{l}40.00(2) \\
60.00(3)\end{array}$ & $\begin{array}{c}0.00(0) \\
100.00(4)\end{array}$ & 0.151 \\
\hline
\end{tabular}

Data expressed as percentage (n) or mean (SD) for categorical or continuous variables, respectively. Abbreviations: BMI, Body Mass Index / Dados expressos em percentagem (n) ou média (desvio padrão) para variáveis categóricas ou contínuas, respetivamente. Abreviaturas: IMC, Índice de Massa Corporal

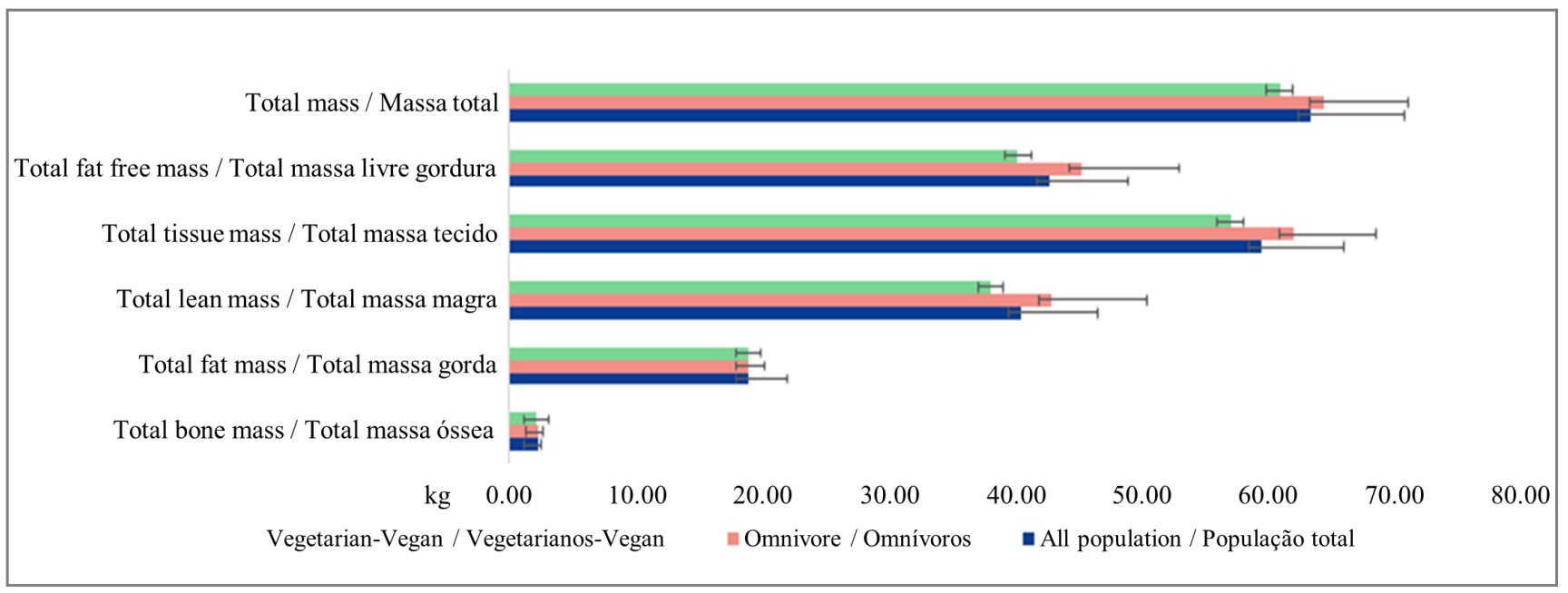

Figure 1 - Body mass distribution according to dietary pattern. Data expressed as mean (SD). $p$-value $>0.05$ Figura 1 - Distribuição de tecido corporal de acordo com o padrão alimentar.Dados expressos como media (desvio padrão). Valor de $p>0.05$. 
Figure 2 shows the areas of the adipose tissue calculation. Values regarding the visceral adipose tissue (VAT) and the subcutaneous adipose tissue (SAT) distribution for the entire studied population are summarized in Table 2, along with a comparison between vegetarianvegan and omnivore participants. The total population presented a mean of $254.10 \mathrm{~cm}^{3}, 240.30 \mathrm{~g}$, and 29.60 $\mathrm{cm}^{2}$ of VAT, and $966.40 \mathrm{~cm}^{3}, 911.60 \mathrm{~g}$, and $109.70 \mathrm{~cm}^{2}$ of SAT. Vegetarian-vegan participants presented higher values of VAT and SAT $\left(281.00\right.$ and $1025.00 \mathrm{~cm}^{3}$, respectively) compared to the omnivore group (219.17 and $831.00 \mathrm{~cm}^{3}$, respectively). None of these differences were statistically significant ( $p$-value $>0.05)$.
A Figura 2 mostra as áreas de cálculo do tecido adiposo. Os valores relativos ao tecido adiposo visceral (TAV) e a distribuição do tecido adiposo subcutâneo (TAS) para toda a população estão resumidos na Tabela 2 , onde também é mostrada a comparação entre os participantes vegetarianos-veganos e omnívoros. A população total apresentou uma média de $254,10 \mathrm{~cm}^{3}, 240,30 \mathrm{~g}$, e $29,60 \mathrm{~cm}^{2}$ de TAV, e também $966,40 \mathrm{~cm}^{3}, 911,60 \mathrm{~g}$, e $109,70 \mathrm{~cm}^{2}$ de TAS. Os participantes vegetarianosveganos apresentaram valores mais elevados de TAV e TAS $\left(281,00\right.$ e $1025,00 \mathrm{~cm}^{3}$, respetivamente) em comparação com os omnívoros $(219,17$ e 831,00 $\mathrm{cm}^{3}$, respetivamente). Nenhuma destas diferenças foi estatisticamente significativa ( $p$-valor $>0,05)$.

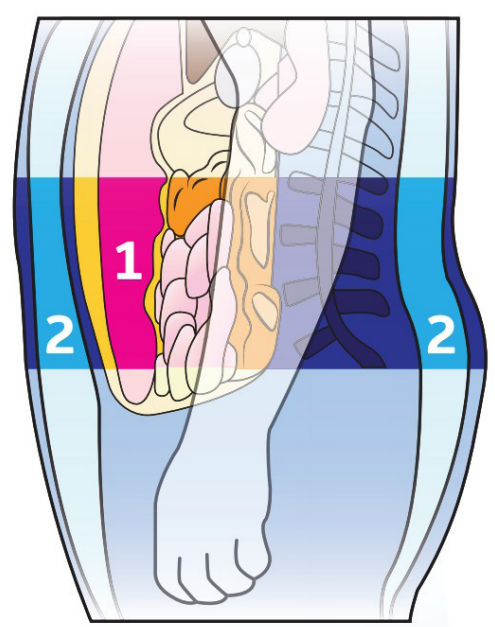

Figure 2 - Schematic representation of tissue distribution of the VAT (1) and the SAT (2) within the android region. [Image: General Electric Company. Reproduced with permission.]

Figura 2 - Representação esquemática da distribuição dos tecidos do TAV (1) e do TAS (2) dentro da região androide. [Imagem: General Electric Company. Reproduzido com permissão.]

Table 2 - Visceral and subcutaneous adipose tissue distribution according to dietary pattern.

Tabela 2 - Distribuição de tecido adipose visceral e subcutâneo de acordo com o padrão alimentar.

\begin{tabular}{|c|c|c|c|c|}
\hline & $\begin{array}{c}\text { Total population / } \\
\text { População total } \\
(\mathrm{n}=10) \\
\end{array}$ & $\begin{array}{c}\text { Omnivore / } \\
\text { Omnívoros } \\
(\mathbf{n}=\mathbf{5}) \\
\end{array}$ & $\begin{array}{c}\text { Vegetarian-Vegan / } \\
\text { Vegetarianos-Vegan } \\
(\mathrm{n}=5) \\
\end{array}$ & $p$-value ${ }^{\mathrm{a}}$ \\
\hline VAT / TAV, $\mathbf{c m}^{3}$ & $254.10(112.38)$ & $222.00(124.75)$ & $286.20(101.38)$ & 0.749 \\
\hline VAT / TAV, grams & $240.30(105.90)$ & $210.20(101.38)$ & $270.40(96.23)$ & 0.742 \\
\hline VAT / TAV, $\mathbf{c m}^{2}$ & $29.60(13.53)$ & $24.80(13.65)$ & $34.40(12.97)$ & 0.651 \\
\hline SAT / TAS, $\mathbf{c m}^{3}$ & $966.40(427.33)$ & $794.40(210.17)$ & $11138.40(210.17)$ & 0.284 \\
\hline SAT / TAS, grams & $911.60(403.28)$ & $749.00(198.33)$ & $1074.20(510.39)$ & 0.284 \\
\hline SAT / TAS, $\mathbf{c m}^{2}$ & $109.70(47.04)$ & $86.60(22.17)$ & $132.80(56.16)$ & 0.250 \\
\hline
\end{tabular}




\section{Discussion}

Obesity and type 2 Diabetes Mellitus (T2DM) represent major risks to develop cardiovascular diseases (CVD), and insulin resistance related to adipose tissue (white and visceral) and pancreatic $\beta$-cells impairment are currently indicated as the primary mechanisms of T2DM (8), these links between obesity, insulin resistance, and CVD have recently drawn more attention to the importance of diet as part of lifestyle and thus a potentially effective way to help prevent these recognized major health burdens, associated or not to physical exercise and activity (9).

Plant-based diets have been described as reducing body fat via a variety of mechanisms, which cumulatively lead to reduced caloric intake and increased energy expenditure (4). These mechanisms might include the reduced caloric density of the overall diet and improved satiety, in part due to gut microbiota alterations (4). Additionally, increased insulin sensitivity, and a potential increase in white adipose tissue. contribute to increasing thermogenesis (4). Plant-based diets have also been consistently associated with reduced body weight in interventional trials $(10,11)$. No matter this very small sample and exploratory character, our results have found no differences in BMI or weight between vegetarian and non-vegetarian participants. No statistical differences were observed regarding the fat mass distribution between those two groups. The vegetarian - vegan group depicted non-statistical higher VAT and SAT values, compared to the omnivore group. In recent years, excess VAT, also referred as visceral obesity. has been recognized as a major predictor of cardiometabolic disease and all-cause mortality independent of BMI and general obesity $(12,13)$. Moreover, VAT is highly metabolically active and contributes to systemic inflammation, and has been assumed to be the common key element and driver of the cardiometabolic risk associated to the metabolic syndrome (14). Data available to date suggests a role for both VAT and SAT in chronic cardiometabolic disease (15). It has been argued that excess abdominal adipose tissue is a consequence of today's modern, sedentary lifestyle patterns, including nutrition and dietary behaviour (13). Inherently, the relationship between dietary intake and health outcomes such as VAT and SAT is very complex, with interactions that are not captured by studying only single dietary components (16).

\section{Discussão}

A obesidade e a Diabetes Mellitus tipo 2 (DM2) representam grandes riscos para o desenvolvimento de doenças cardiovasculares (DCV), sendo atualmente aceite que a resistência à insulina relacionada com o tecido adiposo (branco e visceral) assim como o pâncreas - a deficiência de células $\beta$ - representam os mecanismos primários do DM2 (8). Atualmente, estas ligações entre obesidade, resistência à insulina e DCV têm chamado a atenção para a importância da dieta como parte do estilo de vida e, portanto, uma forma potencialmente eficaz de ajudar a prevenir estes reconhecidos grandes fardos, associados ou não ao exercício físico e à atividade (9).

As dietas à base de plantas foram descritas como uma forma de reduzir a gordura corporal através de uma variedade de mecanismos, que cumulativamente levam à redução do consumo calórico e aumentam o gasto energético (4). Estes mecanismos podem incluir a redução da densidade calórica global da dieta e a melhoria da saciedade, em parte devido a alterações da microbiota intestinal (4). Além disso, o aumento da sensibilidade à insulina, e um potencial aumento do tecido adiposo branco contribuem para aumentar a termogénese (4). As dietas à base de plantas também têm sido consistentemente associadas à redução do peso corporal em ensaios intervencionais $(10,11)$. Independentemente da amostra reduzida e do carácter exploratório do estudo, os nossos resultados não encontraram diferenças estatísticas no IMC ou peso corporal entre os participantes vegetarianos e não vegetarianos. Não foram observadas diferenças estatísticas relativamente à distribuição da massa gorda entre estes dois grupos. O grupo vegetariano-vegan apresentou valores de TAV e TAS não estatisticamente mais elevados, em comparação com o grupo omnívoro. Nos últimos anos, o excesso de TAV também denominado obesidade visceral foi reconhecido como um grande preditor de doenças cardiometabólicas e mortalidade por todas as causas, independentemente do IMC e da obesidade geral $(12,13)$. Além disso, o TAV, que é altamente ativo metabolicamente e contribui para a inflamação sistémica, foi assumido como sendo um elemento chave comum e motor do risco cardiometabólico associado à síndrome metabólica (14). Até à data, os dados disponíveis sugerem um papel tanto do TAV como do TAS na doença cardiometabólica crónica (15). Tem-se argumentado que o excesso de tecido adiposo abdominal é uma consequência dos atuais padrões de vida modernos e sedentários, incluindo nutrição e comportamento dietético (13). 
We might easily agree that no diet, independently, is more healthy than another. In all cases, the diet needs to be properly planned regarding its macro and micronutrient contents and the type of food products chosen (17).

To the best of our knowledge, this is the first published report detecting higher values of VAT and SAT with detailed body mass scanning in vegetarian-vegan young women and compared to a similar omnivore population. The reduced sample is an obvious constraint, limiting our interpretation. Still noninvasive imaging technologies such as DXA are validated to quantify the specific amounts of VAT and SAT in the body (18), and DXA analysis is regarded for that purpose as a "goldstandard". Its accessibility and cost may explain the limited numbers of large scale epidemiological studies using this technique. Since SAT is the biggest reserve of VAT, these results should be further studied with special interest in healthy and normal weight and BMI individuals to better understand its relationship with diet and food group(s) consumption in healthy and nonhealthy individuals.

\section{Conclusion}

This exploratory study revealed some (not statistically significant) differences between healthy volunteers following vegetarian-vegan and omnivorous dietary patterns. Vegetarian-vegan participants have shown consistently lower mass volumes (total bone mass, fat mass, lean mass, tissue mass, and fat-free mass) and higher levels of subcutaneous and visceral adipose tissue when compared with the omnivore group,
Inerentemente, a relação entre o consumo dietético e os resultados de saúde, como o TAV e o TAS, é muito complexa (16).

Poderíamos facilmente concordar que nenhuma dieta é por si só mais saudável do que outras. Em qualquer caso, a dieta precisa de ser devidamente planeada em relação ao seu conteúdo em macro e micronutrientes e ao tipo de produtos alimentares escolhidos (17).

Tanto quanto sabemos, este é o primeiro relatório publicado que deteta valores mais elevados de TAV e TAS com DXA em mulheres jovens vegetarianas e em comparação com uma população omnívora semelhante. A amostra reduzida é um constrangimento óbvio, limitando a nossa interpretação. Tecnologias de imagem ainda não invasivas como o DXA são validadas para quantificar os montantes específicos de TAV e TAS no organismo (18), e a análise DXA é considerada para esse efeito, como um método "padrão dourado". A sua acessibilidade e o seu custo explicam tão poucos estudos epidemiológicos em larga escala utilizando esta técnica. Uma vez que o TAS é a maior reserva de TAV, estes resultados devem ser estudados com especial interesse em indivíduos saudáveis e normoponderais para melhor compreender a sua relação com a dieta e o consumo de grupos alimentares em indivíduos saudáveis e não saudáveis.

\section{Conclusões}

Este estudo exploratório revelou algumas diferenças (não estatisticamente significativas) entre dois grupos dietéticos. Os participantes vegetarianos-veganos mostraram consistentemente volumes de massa mais baixos (massa óssea total, massa gorda, massa magra, massa tecidular e massa sem gordura) e níveis mais elevados de tecido adiposo subcutâneo e visceral quando comparados com o grupo omnívoro. 


\section{Authors Contributions Statement}

C.F.-P., R.G.T, L.M.R conceptualization and study design; R.G.T., S.L., T.F. experimental implementation and data collection; C.F.-P. data analysis; C.F.-P., R.G.T., LMR drafting, editing and reviewing; C.F.-P. figures and graphics; C.F.-P., R.G.T., L.M.R supervision; C.F.-P., R.G.T., L.M.R. final writing.

\section{Funding}

Cíntia Ferreira Pêgo is funded by Foundation for Science and Technology (FCT) Scientific Employment Stimulus contract with the reference number CEEC/ CBIOS/NUT/2018. This work is funded by national funds through FCT - Foundation for Science and Technology, I.P., under the UIDB/04567/2020 and UIDP/ 04567/2020 projects, and ALIES-COFAC PADDICC2021.

\section{Acknowledgements}

The authors acknowledge all the participants.

\section{Conflict of Interests}

The senior editor involved in this manuscript's authorship had no participation in the review or decision process. All authors have stated that there are no financial and/or personal relationships that could represent a potential conflict of interest.

\section{Declaração sobre as contribuições dos autores}

C.F.-P., R.G.T., L.M.R. concetualização e conceção do estudo; R.G.T., S.L., T.F. implementação experimental e recolha de dados; C.F.-P. análise de dados; C.F.- P., R.G.T., L.M.R. redação, edição e revisão; C.F.-P. figuras e gráficos; C.F.-P., R.G.T., L.M.R supervisão; C.F.-P., R.G.T., L.M.R. redação final.

\section{Financiamento:}

Cíntia Ferreira Pêgo é financiada pela Fundação para a Ciência e Tecnologia (FCT) Contrato de Estímulo ao Emprego Científico com o número de referência CEEC/CBIOS/NUT/2018. Este trabalho é financiado por fundos nacionais através da FCT - Fundação para a Ciência e Tecnologia, I.P., ao abrigo dos projetos UIDB/04567/2020 e UIDP/ 04567/2020, e ALIESCOFAC - PADDICC2021.

\section{Agradecimentos}

Os autores agradecem a todos os participantes.

\section{Conflito de Interesses}

O editor senior envolvido na autoria deste manuscrito não tive qualquer participação no processo de revisão ou decisão. Todos os autores declararam que não existem relações financeiras e/ou pessoais que possam representar um potencial conflito de interesses. 


\section{References / Referências}

1. Kaila, B., \& Raman, M. (2008). Obesity: a review of pathogenesis and management strategies. Canadian journal of gastroenterology $=$ Journal canadien de gastroenterologie, 22(1), 61-68. https://doi.org/10.1155/2008/609039.

2. Ratjen, I., Morze, J., Enderle, J., Both, M., Borggrefe, J., Müller, H. P., Kassubek, J., Koch, M., \& Lieb, W. (2020). Adherence to a plant-based diet in relation to adipose tissue volumes and liver fat content. The American journal of clinical nutrition, 112(2), 354-363. https://doi.org/10.1093/ajen/nqaa119

3. Kahleova, H., Levin, S., \& Barnard, N. (2017). Cardio-Metabolic Benefits of Plant-Based Diets. Nutrients, 9(8), 848. https://doi. org/10.3390/nu9080848

4. Najjar, R. S., \& Feresin, R. G. (2019). Plant-Based Diets in the Reduction of Body Fat: Physiological Effects and Biochemical Insights. Nutrients, 11(11), 2712. https://doi.org/10.3390/nu11112712

5. Melina, V., Craig, W., \& Levin, S. (2016). Position of the Academy of Nutrition and Dietetics: Vegetarian Diets. Journal of the Academy of Nutrition and Dietetics, 116(12), 1970-1980. https://doi.org/10.1016/j.jand.2016.09.025

6. Le, L. T., \& Sabaté, J. (2014). Beyond meatless, the health effects of vegan diets: findings from the Adventist cohorts. Nutrients, 6(6), 2131-2147. https://doi.org/10.3390/nu6062131

7. World Medical Association. WMA DECLARATION OF HELSINKI - ETHICAL PRINCIPLES FOR MEDICAL RESEARCH INVOLVING HUMAN SUBJECTS [Internet]. 2018 [cited 2021 Jun 2]. Available from: https://www.wma.net/policies-post/wmadeclaration-of-helsinki-ethical-principles-for-medical-research-involving-human-subjects/

8. Chait, A., \& den Hartigh, L. J. (2020). Adipose Tissue Distribution, Inflammation and Its Metabolic Consequences, Including Diabetes and Cardiovascular Disease. Frontiers in cardiovascular medicine, 7, 22. https://doi.org/10.3389/fcvm.2020.00022

9. Longo, M., Zatterale, F., Naderi, J., Parrillo, L., Formisano, P., Raciti, G. A., Beguinot, F., \& Miele, C. (2019). Adipose Tissue Dysfunction as Determinant of Obesity-Associated Metabolic Complications. International journal of molecular sciences, $20(9), 2358$. https://doi.org/10.3390/ijms20092358

10. Barnard, N. D., Levin, S. M., \& Yokoyama, Y. (2015). A systematic review and meta-analysis of changes in body weight in clinical trials of vegetarian diets. Journal of the Academy of Nutrition and Dietetics, 115(6), 954-969. https://doi.org/10.1016/j.jand.2014.11.016.

11. Huang, R. Y., Huang, C. C., Hu, F. B., \& Chavarro, J. E. (2016). Vegetarian Diets and Weight Reduction: a Meta-Analysis of Randomized Controlled Trials. Journal of general internal medicine, 31(1), 109-116. https://doi.org/10.1007/s11606-015-3390-7

12. Kuk, J. L., Katzmarzyk, P. T., Nichaman, M. Z., Church, T. S., Blair, S. N., \& Ross, R. (2006). Visceral fat is an independent predictor of all-cause mortality in men. Obesity (Silver Spring, Md.), 14(2), 336-341. https://doi.org/10.1038/oby.2006.43

13. Tchernof, A., \& Després, J. P. (2013). Pathophysiology of human visceral obesity: an update. Physiological reviews, 93(1), 359-404. https://doi.org/10.1152/physrev.00033.2011

14. Ibrahim M. M. (2010). Subcutaneous and visceral adipose tissue: structural and functional differences. Obesity reviews : an official journal of the International Association for the Study of Obesity, 11(1), 11-18. https://doi.org/10.1111/j.1467-789X.2009.00623.x

15. Abate, N., \& Chandalia, M. (2012). Role of subcutaneous adipose tissue in metabolic complications of obesity. Metabolic syndrome and related disorders, 10(5), 319-320. https://doi.org/10.1089/met.2012.1502

16. Fischer, K., Pick, J. A., Moewes, D., \& Nöthlings, U. (2015). Qualitative aspects of diet affecting visceral and subcutaneous abdominal adipose tissue: a systematic review of observational and controlled intervention studies. Nutrition reviews, 73(4), 191-215. https://doi. org/10.1093/nutrit/nuu006

17. Kahleova, H., Petersen, K. F., Shulman, G. I., Alwarith, J., Rembert, E., Tura, A., Hill, M., Holubkov, R., \& Barnard, N. D. (2020). Effect of a Low-Fat Vegan Diet on Body Weight, Insulin Sensitivity, Postprandial Metabolism, and Intramyocellular and Hepatocellular Lipid Levels in Overweight Adults: A Randomized Clinical Trial. JAMA network open, 3(11), e2025454. https://doi.org/10.1001/ jamanetworkopen.2020.25454/

18. Shuster, A., Patlas, M., Pinthus, J. H., \& Mourtzakis, M. (2012). The clinical importance of visceral adiposity: a critical review of methods for visceral adipose tissue analysis. The British journal of radiology, 85(1009), 1-10. https://doi.org/10.1259/bjr/38447238 